\title{
Impulsividad en la Adolescencia: Utilización de una Versión Breve del Cuestionario UPPS en una Muestra de Jóvenes Latinoamericanos y Españoles
}

\author{
Impulsivity in Adolescence: Use of a Short Version of the UPPS Questionnaire in a \\ Sample of Latin American and Spanish Youths
}

\author{
Paulina Herdoiza-Arroyo ${ }^{1}$ y Mariano Chóliz ${ }^{2}$
}

\begin{abstract}
Resumen
La impulsividad es un constructo multidimensional, vinculado con comportamientos de riesgo en adolescentes. Algunos estudios señalan que durante este período, la impulsividad varía en niveles y dimensiones. Sin embargo, dichos estudios son escasos, muestran resultados mixtos, o exploran pocas facetas de la impulsividad. En el presente trabajo, de diseño transversal, fueron examinadas las dimensiones de impulsividad mediante una versión corta del cuestionario UPPS, en relación con edad y género. Participaron 2,055 adolescentes de 11 a 17 años de Latinoamérica y España. En las comparaciones por género, Búsqueda de sensaciones y Urgencia mostraron diferencias significativas. En las comparaciones por edad y género, las mujeres presentaron diferencias significativas en Urgencia, Búsqueda de sensaciones y Perseverancia, mientras que los varones mostraron tales diferencias sólo en Urgencia. Estos hallazgos proporcionan información relevante para el desarrollo de programas de prevención o intervención en comportamientos de riesgo de adolescentes asociados con la impulsividad.
\end{abstract}

Palabras clave: impulsividad, adolescencia, UPPS, género

\begin{abstract}
Impulsivity is a multidimensional construct, linked to risk behaviors in adolescents. Some studies have shown that during this period impulsivity varies in levels and dimensions. However, such studies are scarce, show mixed results, or explore few facets of impulsivity. In this cross-sectional study, the dimensions of impulsivity in relation to age and gender were examined using a short version of the UPPS questionnaire. A total of 2,055 adolescents, aged 11 to 17, from Latin America and Spain, participated in the study. In the comparisons by gender, Sensation Seeking and Urgency showed significant differences. In the comparisons by age and gender, females presented significant differences in Urgency, Sensation Seeking, and Perseverance, while males showed such differences only in Urgency. These findings provide relevant information for the development of prevention or intervention programs in adolescent risk behaviors associated with impulsivity.
\end{abstract}

Keywords: impulsivity, adolescence, UPPS, gender

\footnotetext{
${ }^{1}$ Máster Universitario. Estudiante de PhD, Universidad de Valencia, Facultat de Psicologia, Avinguda Blasco Ibáñez, 21. CP. 46010. Valencia, España. Profesora Titular, Universidad Internacional del Ecuador, Avenida Simón Bolívar y Avenida Jorge Fernández, Quito, Ecuador. Tel.: +5932 298 5600, extensión 6031. Correo: pherdoiza@uide.edu.ec

${ }^{2} \mathrm{PhD}$, Universidad de Valencia, Facultat de Psicologia, Avinguda Blasco Ibáñez, 21. CP. 46010. Valencia, España. Tel.: 003496398 3579. Correo: mariano.choliz@uv.es
} 


\section{Introducción}

La impulsividad es un constructo multidimensional y heterogéneo, que ha dado lugar a múltiples conceptualizaciones. Es así que se ha definido como aquellas acciones que se expresan sin antes haber sido meditadas lo suficiente, que suponen riesgo o son inapropiadas para la situación y que suelen acarrear consecuencias indeseables (Durana \& Barnes, 1993). Se ha distinguido entre impulsividad atencional, impulsividad motora e impulsividad sin planificación (Patton, Stanford, \& Barratt, 1995), o entre elección impulsiva y acción impulsiva (Stevens, 2017), entre otras aproximaciones conceptuales. En este panorama de multiplicidad de aspectos, etiquetados como impulsividad (Cyders, 2015), surgió el cuestionario UPPS (Whiteside \& Lynam, 2001) como un esfuerzo por clarificar las dimensiones que conforman este complejo constructo. Whiteside y Lynam (2001) identificaron cuatro dimensiones, a saber, Urgencia, relacionada con la conducta impulsiva que surge como reacción ante un intenso afecto negativo; (Falta de) Premeditación, que implica la habilidad para elegir una opción tomando en cuenta las posibles consecuencias; (Falta de) Perseverancia, que evalúa la habilidad para permanecer en las tareas, especialmente, si son difíciles o aburridas; y Búsqueda de sensaciones que se refiere a la tendencia a buscar actividades nuevas o que suponen riesgo. Estas dimensiones permitieron comprender la impulsividad desde sus aspectos de carácter emocional/ afectivo (en Urgencia y Búsqueda de sensaciones), así como desde vertientes más cognitivas (en Perseverancia y Premeditación).

El papel de estas diferentes dimensiones de impulsividad en conductas problemáticas o de riesgo ha sido objeto de interés científico en trabajos realizados con adolescentes, mediante el UPPS (Whiteside \& Lynam, 2001), el UPPS-P (Cyders et al., 2007); este último, incluye la dimensión de Urgencia positiva, o el UPPS-R-C (Zapolski, Stairs, Settles, Combs, \& Smith, 2010), validado con niños. Solamente en algunos de dichos trabajos se ha tenido en cuenta la variable de género para la exploración de estas dimensiones. Tal es el caso de un estudio sobre conductas antisociales y su vínculo con la impulsividad (Maneiro, Gómez-Fraguela, Cutrín, Romero, 2017), en el cual se identificó que únicamente en la dimensión de Búsqueda de sensaciones las diferencias entre varones $y$ mujeres fueron significativas, siendo los primeros quienes presentaron valores más altos. En cuanto a Urgencia negativa, las mujeres obtuvieron una media más alta que la de los varones, aunque dicha diferencia de medias no fue significativa. Por otra parte, en el mismo estudio, las dimensiones de impulsividad que correlacionaron de forma positiva y significativa con todas las conductas antisociales evaluadas (agresión, ruptura de reglas, robo y vandalismo) fueron Urgencia positiva, Urgencia negativa y Búsqueda de sensaciones. Adicionalmente, Falta de premeditación correlacionó positivamente con vandalismo, y Falta de perseverancia con robo y vandalismo.

El papel de las dimensiones de Urgencia, tanto positiva como negativa, también se observó en el uso problemático de alcohol y cannabis en adolescentes (Stautz \& Cooper, 2014; Tomko, Prisciandaro, Falls, \& Magid, 2016). En ambos trabajos no realizaron comparaciones por sexos, debido al pequeño tamaño muestral, y a que la mayoría de participantes eran, respectivamente, varones (Tomko et al., 2016) y mujeres (Stautz \& Cooper, 2014). Estos resultados van en consonancia con los encontrados en el metaanálisis, realizado por Stautz y Cooper (2013), en el cual ambos tipos de Urgencia fueron las facetas de impulsividad que presentaron vínculos más fuertes con problemas derivados del uso excesivo de alcohol.

Las dimensiones de impulsividad se han visto relacionadas de forma positiva y significativa con otras problemáticas infanto- juveniles, además de las mencionadas anteriormente. Así, por ejemplo, en otro trabajo (Zapolski et al., 2010), la conducta agresiva se vinculó con Urgencia negativa; los problemas atencionales con Falta de perseverancia y Falta de premeditación; las conductas altamente emocionantes que pueden implicar riesgo, con Búsqueda de sensaciones y con Falta de premeditación; las conductas de riesgo ante estados de ánimo intensamente negativos, con Urgencia negativa y Falta de premeditación; y el desempeño académico, con Falta de perseverancia 
y Falta de premeditación. En el mencionado estudio, dado que la mayoría de participantes eran varones, no se estudiaron las diferencias entre géneros.

Otro ámbito desde el que se ha estudiado la impulsividad en la adolescencia es a través del desarrollo. Evidencia procedente de estudios recientes, tanto longitudinales como transversales, indica ciertos patrones diferenciales en las trayectorias evolutivas de las distintas facetas de impulsividad, que estarían en consonancia con el modelo de sistemas duales (Casey, Getz, \& Galvan, 2008), modelo que postula que en la adolescencia, como un aspecto normativo e inherente al desarrollo cerebral, hay un desajuste entre el sistema emocional o afectivo, -que está bastante activo en la etapa adolescente-, y el sistema de control o gestión de la conducta, -que se halla todavía pendiente de desarrollar, y que irá regulando, progresivamente, la impulsividad de tipo emocional-.

En este sentido, estudios indican que Búsqueda de sensaciones presenta una trayectoria de crecimiento lineal a lo largo de la adolescencia, que sitúa a la adolescencia media como el período del ciclo vital en el que se acoge con mayor interés el experimentar situaciones altamente emocionantes; más, incluso, que en otros momentos de la adolescencia (Collado, Felton, MacPherson, \& Lejuez, 2014; Harden \& TuckerDrob, 2011; Steinberg et al., 2008) o de la adultez (Harden \& Tucker-Drob, 2011; Steinberg et al., 2008). Mientras que, en los mencionados estudios, la Impulsividad presenta una trayectoria de declive progresivo a partir de la adolescencia media, que continúa a medida que avanza la edad.

Varios trabajos realizados con adolescentes, si bien aportan información relevante, presentan algunas limitaciones; por ejemplo, en cuanto a los instrumentos de medida utilizados. Tal es el caso del estudio de Harden y Tucker-Drob (2011) en el cual evaluaron los cambios longitudinales ocurridos en Impulsividad y en Búsqueda de sensaciones mediante únicamente seis ítems. Otras de las limitaciones encontradas en algunos estudios son que no realizaron comparaciones por sexos en las muestras de adolescentes (Harden \& Tucker-Drob, 2011) o que los resultados, al llevar a cabo dichas comparaciones, fueron mixtos: estudios que señalan perfiles similares entre mujeres y varones (por ejemplo, Collado et al., 2014; MacPherson, Magidson, Reynolds, Kahler, \& Lejuez, 2010) frente a otros que sí identificaron diferencias por sexos (Pedersen, Molina, Belendiuk, \& Donovan, 2012; Romer \& Hennessy, 2007, Steinberg et al., 2008). A este respecto, conviene mencionar que en el estudio de Steinberg et al. (2008) no encontraron diferencias significativas entre los sexos al analizar la muestra completa, aunque sí las hallaron al comparar submuestras de 16 años o menos, en Búsqueda de sensaciones. Asimismo, se reportan en los mencionados estudios solamente algunas de las facetas de impulsividad, concretamente, Búsqueda de sensaciones y una variable etiquetada como Impulsividad (que contempla control cognitivo de la conducta, aunque su operacionalización varía, según estudios), pero no se exploran dimensiones como, por ejemplo, la Urgencia o la Falta de perseverancia (Whiteside \& Lynam, 2001). Pese a que la Urgencia negativa guarda fuertes relaciones con conductas problemáticas en la adolescencia (Stautz \& Cooper, 2014) ha sido una dimensión menos estudiada, desde una perspectiva del desarrollo.

Hasta donde conocemos, solamente un estudio (Littlefield, Stevens, Ellingson, King, \& Jackson, 2016) ha utilizado el UPPS-P en una muestra de adolescentes desde una aproximación evolutiva. Los hallazgos de dicho trabajo longitudinal sugieren que la Urgencia negativa sigue una trayectoria similar a la evidenciada por Urgencia positiva y por Búsqueda de sensaciones, con un aumento en los niveles en torno a los 11 a 13 años, y su estabilización en edades posteriores. Littlefield et al. (2016), no obstante, no estudiaron los demás componentes de la impulsividad recogidos por el UPPS, a saber, aquellos de carácter más cognitivo: Perseverancia y Premeditación.

Aunque varios estudios arrojan evidencia que sustenta que Urgencia negativa y Urgencia positiva son dos dimensiones separadas (por ejemplo, Cyders \& Smith, 2007, 2008), y que cada una de ellas está vinculada de forma específica con determinados trastornos o conductas problemáticas, algunos trabajos recientes reportan fuertes similitudes entre ambas facetas, que en su conjunto conforman un rasgo de personalidad o un endofenotipo transdiagnóstico 
(Cyders, Coskunpinar, \& VanderVeen, 2016), presente en trastornos que implican la participación en conductas de riesgo. $\mathrm{La}$ semejanza entre las trayectorias de Urgencia positiva y Urgencia negativa (Littlefield et al., 2016) va en la misma línea de resultados de otros trabajos, que señalan que las dos dimensiones de Urgencia, con independencia de la valencia afectiva a la que hacen referencia, demuestran convergencias, por ejemplo, en los patrones correlacionales con diferentes psicopatologías (Berg et al., 2015), en el vínculo que ambas reflejan con el afecto negativo (Borges, Dahne, Lim, \& MacPherson, 2017; Sperry, Lynam \& Kwapil, 2017), así como en su nexo común con el neuroticismo (Cyders \& Smith, 2007; Whiteside \& Lynam, 2001). Esta estrecha relación entre las dos dimensiones se aprecia también en los valores de inter correlación entre ambas (que arroja, por ejemplo, datos de $\mathrm{r}=.77$, en el estudio de Sperry et al., 2017, o de $\mathrm{r}=.70$ en el trabajo de Borges et al., 2017), o en el elevado coeficiente alfa obtenido al integrar los ítems de ambas Urgencias en una sola escala (coeficiente alfa $=.93$ en Sperry et al., 2017, o coeficiente alfa $=.91$ en Burris, Riley, Puleo, \& Smith, 2017). Estos hallazgos sugieren que las Urgencias conforman dos procesos íntimamente ligados y en ocasiones solapados entre sí, cuya separación suscita interrogantes en torno a la pertinencia conceptual y práctica de asumirlos como dos aspectos diferentes de la impulsividad, como se reporta en la revisión meta- analítica conducida por Berg et al. (2015), así como en el trabajo sobre convergencias y divergencias entre facetas de impulsividad en la vida diaria realizado por Sperry et al. (2017), quienes sugieren la conformación de un único factor de Urgencia en lugar de dos. Por otra parte, en el mencionado trabajo de meta- análisis, los autores (Berg et al., 2015) señalan que, de las dos dimensiones de Urgencia, fue la Urgencia negativa la que se identificó como más fuertemente implicada en el desarrollo de la conducta impulsiva, al presentar tamaños del efecto correlacionales mayores que los obtenidos en las demás dimensiones del UPPS/UPPS-P en un amplio rango de síntomas psicopatológicos (rasgos de personalidad limítrofe, trastornos de la alimentación, conductas autolesivas, agresión y psicopatía, depresión, ansiedad); de hecho, solamente en el uso de alcohol y de sustancias, la Urgencia positiva tuvo un efecto de similar magnitud. De ahí que dichos autores manifiestan que el estudio de la Urgencia negativa podría ser clave para obtener la mayor información sobre la impulsividad en relación con diferentes tipos de psicopatología (Berg et al., 2015).

A la luz de los hallazgos antes mencionados, en el presente estudio se utilizó una versión breve del UPPS, que contempla únicamente la Urgencia negativa, dada la importancia de esta faceta en un amplio rango de conductas desadaptativas y psicopatológicas, así como debido a las fuertes similitudes que esta guarda con la Urgencia positiva.

Los objetivos del presente estudio fueron: a) Explorar las dimensiones de impulsividad, mediante el UPPS, en una muestra amplia de adolescentes de ambos sexos, procedentes de América Latina y España, b) Identificar si existen diferencias significativas por sexos en las dimensiones de impulsividad, c) Analizar las trayectorias evolutivas por edades, desde la adolescencia temprana hasta la adolescencia media, pasando por una etapa de transición entre ambas d) Valorar si las trayectorias evolutivas por edades difieren al comparar a los participantes por sexos.

Planteamos como hipótesis que en este estudio se replicará la estructura factorial de cuatro componentes, factores que irán en consonancia con los planteados por los autores del UPPS y replicados en otros trabajos; b) Habrá diferencias significativas en los niveles de Búsqueda de sensaciones, los cuales serán mayores para varones que para mujeres, en consonancia con los resultados de estudios que han empleado el UPPS, así como otros instrumentos. No se identificarán diferencias significativas entre sexos en las dimensiones de impulsividad de corte más cognitivo (Premeditación y Perseverancia), tal como lo evidencian estudios previos. En cuanto a Urgencia, no podemos establecer una hipótesis clara en torno a diferencias entre los sexos, puesto que la evidencia al respecto presenta resultados mixtos; c) Habrá diferencias significativas por edades, y la adolescencia media será la etapa que presente mayores niveles de impulsividad, en todas las dimensiones; d) No podemos establecer 
una hipótesis clara sobre las trayectorias evolutivas por edades comparando por sexos ya que los estudios al respecto son escasos y presentan resultados mixtos.

\section{Método}

\section{Participantes}

Formaron parte del estudio 2211 adolescentes de entre 9 y 19 años, que estuvieran escolarizados en sus respectivas unidades educativas. Del total de la muestra se excluyó a un $7 \%$ de los participantes $(\mathrm{n}=156)$ debido a presentar una edad inferior a 11 o superior a 17 años. Por tanto, la muestra final estuvo conformada por 2055 jóvenes de entre 11 y 17 años (51.7\% fueron varones). Cabe mencionar que $0.3 \%$ de la muestra $(n=7)$ no indicó su género. Estos participantes, no obstante, fueron retenidos para las comparaciones por edad. La media de edad grupal fue 13.94 años (DT=1.75); la media de los varones fue 13.99 años (DT=1.74) y la de las mujeres, 13.91 años (DT=1.76). La diferencia entre ambas medias de edad no fue significativa $(\mathrm{t}(2046)=.995, p=.320$, $\mathrm{d}=.05,95 \%$ IC $[-.08, .23])$. Los adolescentes procedieron de países latinoamericanos (Ecuador $32 \%$, México 25\%, Perú 22\%), y de España $(21 \%)$.

\section{Instrumentos}

Los participantes cumplimentaron a manera de autoinforme una versión corta del UPPS original (Whiteside \& Lynam, 2001), versión desarrollada por Keye, Wilhelm y Oberauer (2009). Esta versión corta está conformada por 20 ítems, presentados en una escala tipo Likert de cinco puntos $(1=$ Totalmente en Desacuerdo, 5= Totalmente de Acuerdo), distribuidos en los mismos cuatro factores de la escala original: Urgencia, (Falta de) Premeditación, (Falta de) Perseverancia, y Búsqueda de sensaciones. Los valores de alfa de Cronbach obtenidos por Keye et al. (2009) fueron: Premeditación .75, Urgencia .74, Búsqueda de sensaciones .72 y Perseverancia .75. Los ítems recogidos en dicho estudio (Keye et al., 2009) se encontraban en alemán e inglés. Para el presente trabajo se realizó una comprobación de la equivalencia de significado de cada uno de los ítems, con respecto de la versión original.

\section{Procedimiento}

Mediante un muestreo no probabilístico, por conveniencia, se contactó con diversos centros educativos de nivel medio, a través de correo electrónico o por vía telefónica. Con aquellos que se interesaron por el estudio se estableció una comunicación personal para explicar detalladamente los objetivos del mismo, coordinar el permiso y gestionar el consentimiento informado dirigido a los padres de familia o representantes, así como a los estudiantes.

Esta investigación fue desarrollada según estándares éticos recogidos por la American Psychological Association (2010). Se explicaron por escrito y de forma verbal los objetivos del estudio, así como en qué consistiría la participación, el carácter voluntario de la misma, el anonimato, y la utilización confidencial de los datos. La administración del cuestionario se llevó a cabo en una sola sesión, dentro de la jornada escolar ordinaria diurna, durante 20 minutos. Todos los grupos de estudiantes fueron evaluados, de forma simultánea, en sus aulas naturales, dentro de cada centro educativo. Los participantes cumplimentaron el cuestionario a manera de autoinforme, tras unas indicaciones breves. Durante el pase del cuestionario, en cada una de las aulas, se encontró un experto para responder posibles dudas sobre el estudio. Los datos obtenidos fueron analizados mediante el programa IBM SPSS Statistics 22. El análisis de los valores perdidos reveló que en ninguno de los ítems había una cantidad de dichos valores superior al $0.8 \%$; no obstante, se realizó una imputación de estos por el valor medio de la subescala correspondiente a la que pertenece dicho ítem. Los resultados obtenidos con los datos imputados y sin imputar fueron muy similares, y se decidió realizar los análisis posteriores teniendo en cuenta los imputados. Se efectuaron pruebas de fiabilidad, análisis factorial exploratorio, análisis de varianza y comparaciones post hoc.

\section{Resultados}

El valor de alfa de Cronbach para la escala completa fue de .73. ( $\mathrm{T}$ cuadrado de Hotelling $=2669.51, \mathrm{~F}(19,2036)=139.27, p<.001)$. 
Tabla 1. Análisis factorial exploratorio con rotación Varimax del UPPS breve, tras suprimir el ítem 11

\begin{tabular}{|c|c|c|c|c|}
\hline \multirow{2}{*}{ Ítems } & \multicolumn{4}{|c|}{ Componente } \\
\hline & I & II & III & IV \\
\hline Ítem $15 \mathrm{~A}$ veces hago cosas impulsivamente de las que después me arrepiento. & .65 & .06 & .31 & -.18 \\
\hline Ítem $01 \mathrm{Me}$ resulta difícil controlar mis impulsos (hago las cosas sin reflexionar). & .63 & .05 & .02 & -.05 \\
\hline $\begin{array}{l}\text { Ítem } 12 \text { Cuando me encuentro triste o desanimado suelo hacer cosas que no debería } \\
\text { hacer. }\end{array}$ & .62 & .05 & -.08 & -.13 \\
\hline Ítem 10 Hay tantas pequeñas tareas por hacer que a veces me desentiendo de todas. & .61 & .07 & -.22 & .06 \\
\hline $\begin{array}{l}\text { Ítem } 20 \text { En el acaloramiento de una discusión digo cosas de las que después me } \\
\text { arrepiento. }\end{array}$ & .58 & .03 & .37 & -.14 \\
\hline Ítem 05 Suelo abandonar o rendirme ante las tareas fácilmente. & .56 & .09 & -.36 & .06 \\
\hline $\begin{array}{l}\text { Ítem } 09 \text { Cuando estoy triste o desanimado, no puedo dejar de hacer lo que estoy } \\
\text { haciendo en ese momento, aunque después me sienta peor. }\end{array}$ & .56 & .03 & -.02 & .22 \\
\hline Ítem 14 Disfrutaría saltando en paracaídas o en parapente. & .06 & .77 & .12 & .02 \\
\hline Ítem 07 Me gustaría pilotar una avioneta. & .04 & .75 & .04 & .06 \\
\hline $\begin{array}{l}\text { Ítem } 18 \text { Disfrutaría bajando a toda velocidad con esquís por la ladera de una } \\
\text { montaña. }\end{array}$ & .12 & .73 & .12 & .02 \\
\hline Ítem 02 Me gustaría hacer buceo submarino. & .03 & .65 & .11 & .05 \\
\hline Ítem 16 Cuando empiezo algo, casi siempre lo acabo. & .00 & .10 & .70 & .13 \\
\hline Ítem 13 Suelo acabar lo que comienzo. & -.13 & .14 & .66 & .26 \\
\hline Ítem 17 Antes de comenzar una actividad nueva me gusta saber cosas de ella. & .04 & .12 & .57 & .25 \\
\hline Ítem 06 Ir a mi ritmo me permite acabar las cosas bien y a tiempo. & .02 & .13 & .43 & .25 \\
\hline Ítem 04 Generalmente planifico detenidamente antes de hacer cualquier cosa. & -.07 & .01 & .22 & .67 \\
\hline Ítem $03 \mathrm{Me}$ controlo, es decir, no "suelto" o digo las cosas sin pensar. & .06 & .08 & .00 & .64 \\
\hline Ítem 19 Generalmente razono con calma antes de tomar las decisiones & -.11 & .02 & .33 & .60 \\
\hline Ítem $08 \mathrm{Me}$ gusta detenerme a pensar antes de actuar. & .00 & .03 & .31 & .60 \\
\hline
\end{tabular}

Nota. Saturaciones factoriales $>.40$ en negrita. Componentes: I Urgencia, II Búsqueda de sensaciones, III Perseverancia, IV Premeditación

Se realizó el análisis factorial exploratorio con rotación Varimax, y se encontró que el ítem 11 ("Generalmente busco sensaciones y experiencias que sean nuevas y excitantes") presentó saturaciones moderadas en dos factores, concretamente en Búsqueda de sensaciones, del cual es originario (.38), y en Urgencia (.36). Por tanto, se decidió eliminar este ítem para los análisis posteriores. Se calculó nuevamente el alfa de Cronbach que fue de .72 para la escala completa ( $\mathrm{T}$ cuadrado de Hotelling $=2568.76$, $\mathrm{F}(18, \quad 2037)=146.49, \quad p<.001), \quad \mathrm{y}$ para las respectivas dimensiones de impulsividad fue de la siguiente manera: Urgencia .72, Búsqueda de sensaciones .72, Perseverancia .64 y Premeditación .63. Cabe mencionar que al calcular alfa de Cronbach sin el ítem 11, la fiabilidad de Búsqueda de sensaciones aumentó ligeramente (pasó de .70 a .72). Los resultados recabados, para grupo completo, en la medida de adecuación muestral de Kaiser-Meyer-Olkin (KMO=.81), y la prueba de esfericidad de Bartlett (Chi cuadrado $(171)=7013.64, p<.001)$ revelan que el modelo factorial es adecuado para explicar los datos.

La Tabla 1 recoge los ítems y sus saturaciones en la matriz resultante. La estructura factorial refleja los cuatro factores originales, que explican, en total, el $46.98 \%$ de la varianza: el primero corresponde con Urgencia, y explica el $17.47 \%$ de la varianza; el segundo representa Búsqueda de sensaciones y explica $14.80 \%$ de la varianza; el tercero refleja Perseverancia y explica $8.84 \%$ de la varianza, y el cuarto componente recoge los ítems de Premeditación y explica 5.87\% de la varianza. Si bien la mayoría de los ítems se mantuvieron en su factor original, tres de ellos migraron a otro factor. Tal es el caso de los ítems 5 y 10, inicialmente de Perseverancia migrados a Urgencia, y del ítem 17, originalmente de Premeditación migrado a Perseverancia. Se conformaron, por tanto, los factores de Urgencia 
Tabla 2. Comparaciones por género en las dimensiones del UPPS

\begin{tabular}{lccc}
\hline Dimensión & Varones & Mujeres & Todos \\
& $\mathrm{n}=1061$ & $\mathrm{n}=987$ & $\mathrm{~N}=2048$ \\
$\mathrm{M}(\mathrm{DT})$ & $\mathrm{M}(\mathrm{DT})$ & $19.46(5.45)$ & $19.07(5.46)$ \\
\hline Urgencia & $18.75(5.43)$ & $13.09(4.36)$ & $13.80(4.43)$ \\
Búsqueda de sensaciones & $14.48(4.39)$ & $14.55(3.22)$ & $14.56(3.30)$ \\
Perseverancia & $14.56(3.36)$ & $13.33(3.21)$ & $13.29(3.31)$ \\
Premeditación & $13.25(3.39)$ & $52.66(9.27)$ & $53.02(8.97)$ \\
Impulsividad Total & $53.41(8.66)$ &
\end{tabular}

Tabla 3. Comparaciones por edad para grupo completo. ANOVA y Comparaciones Post hoc

\begin{tabular}{|c|c|c|c|c|c|c|c|}
\hline \multirow[b]{2}{*}{ Dimensión } & \multicolumn{3}{|c|}{ Todos } & \multicolumn{4}{|r|}{ ANOVA } \\
\hline & $\begin{array}{c}\text { G 1 } \\
\text { M (DT) }\end{array}$ & $\begin{array}{c}\text { G } 2 \\
M \text { (DT) }\end{array}$ & $\begin{array}{c}\text { G 3 } \\
\text { M (DT) }\end{array}$ & $\mathrm{F}$ & $p$ & $\eta p^{2}$ & $\begin{array}{c}\text { Comparaciones } \\
\text { Tukey HSD }\end{array}$ \\
\hline Urgencia & $18.19(6.06)$ & $19.76(5.51)$ & $19.10(4.88)$ & 12.835 & .000 & .012 & $\mathrm{G} 1<\mathrm{G} 2 * * * \mathrm{G} 1<\mathrm{G} 3 * * \mathrm{G} 3<\mathrm{G} 2 *$ \\
\hline $\begin{array}{l}\text { Búsqueda de } \\
\text { Sensaciones }\end{array}$ & $13.42(4.68)$ & $13.85(4.58)$ & $14.02(4.11)$ & 3.098 & .045 & .003 & $\mathrm{G} 1<\mathrm{G} 3 *$ \\
\hline Perseverancia & $14.95(3.57)$ & $14.40(3.43)$ & $14.43(2.97)$ & 5.404 & 005 & .005 & $\mathrm{G} 1>\mathrm{G} 2 * * \quad \mathrm{G} 1>\mathrm{G} 3 *$ \\
\hline Premeditación & $13.22(3.72)$ & $13.07(3.42)$ & $13.52(2.89)$ & 3.601 & .027 & .003 & $\mathrm{G} 2<\mathrm{G} 3 *$ \\
\hline Impulsividad Total & $51.44(9.64)$ & $54.14(9.47)$ & $53.17(7.87)$ & 14.194 & .000 & .014 & $\mathrm{G} 1<\mathrm{G} 2 * * * \mathrm{G} 1<\mathrm{G} 3 * *$ \\
\hline
\end{tabular}

Nota. G1=11 y 12 años, G2=13 y 14 años, G3=15 a 17 años. Valores estadísticamente significativos del ANOVA en negrita Grados de libertad entre grupos $=2$, intra grupos $=2052$. En comparaciones Tukey $* p<.05$, $* * p<.01, * * * p<.001$

Tabla 4. Estadísticos de los varones por edades. ANOVA y Comparaciones Post hoc

\begin{tabular}{|c|c|c|c|c|c|c|c|}
\hline \multirow[b]{2}{*}{ Dimensión } & \multicolumn{3}{|c|}{ Varones } & \multirow[b]{2}{*}{$\mathrm{F}$} & \multirow[b]{2}{*}{$p$} & \multicolumn{2}{|c|}{ ANOVA } \\
\hline & $\begin{array}{c}\text { G 1 } \\
\text { M (DT) }\end{array}$ & $\begin{array}{c}\text { G } 2 \\
\text { M (DT) }\end{array}$ & $\begin{array}{c}\text { G 3 } \\
\text { M (DT) }\end{array}$ & & & $\eta p^{2}$ & $\begin{array}{c}\text { Comparaciones } \\
\text { Tukey HSD } \\
\end{array}$ \\
\hline Urgencia & $18.01(6.13)$ & $19.34(5.56)$ & $18.72(4.77)$ & 4.703 & .009 & .009 & $\mathrm{G} 1<\mathrm{G} 2 * *$ \\
\hline $\begin{array}{l}\text { Búsqueda de } \\
\text { Sensaciones }\end{array}$ & $14.31(4.69)$ & $14.68(4.45)$ & $14.42(4.14)$ & .603 & .547 & .001 & \\
\hline Perseverancia & $14.68(3.81)$ & $14.60(3.47)$ & $14.45(2.95)$ & .434 & 648 & .001 & \\
\hline Premeditación & $13.12(3.83)$ & $13.04(3.57)$ & $13.51(2.91)$ & 2.169 & .115 & .004 & \\
\hline Impulsividad Total & $52.51(9.28)$ & $54.37(9.43)$ & $53.18(7.43)$ & 3.840 & .022 & .007 & $\mathrm{G} 1<\mathrm{G} 2 *$ \\
\hline
\end{tabular}

Nota. G1=11 y 12 años, G2=13 y 14 años, G3=15 a 17 años. Valores estadísticamente significativos del ANOVA en negrita

Grados de libertad entre grupos $=2$, intra grupos $=1058$. En comparaciones Tukey $* p<.05, * * p<.01$

Tabla 5. Estadísticos de las mujeres por edades. ANOVA y Comparaciones Post hoc

\begin{tabular}{|c|c|c|c|c|c|c|c|}
\hline \multirow[b]{2}{*}{ Dimensión } & \multicolumn{5}{|c|}{ Mujeres } & \multicolumn{2}{|r|}{ ANOVA } \\
\hline & $\begin{array}{c}\text { G 1 } \\
\text { M (DT) }\end{array}$ & $\begin{array}{c}\text { G 2 } \\
M(D T)\end{array}$ & $\begin{array}{c}\text { G 3 } \\
\text { M (DT) }\end{array}$ & $\mathrm{F}$ & $p$ & $\eta p^{2}$ & $\begin{array}{c}\text { Comparaciones } \\
\text { Tukey HSD }\end{array}$ \\
\hline Urgencia & $18.46(5.99)$ & $20.25(5.41)$ & $19.51(4.97)$ & 8.098 & .000 & .016 & $\mathrm{G} 1<\mathrm{G} 2 * * * \mathrm{G} 1<\mathrm{G} 3 *$ \\
\hline $\begin{array}{l}\text { Búsqueda de } \\
\text { Sensaciones }\end{array}$ & $12.57(4.50)$ & $12.93(4.56)$ & $13.58(4.04)$ & 4.718 & .009 & .009 & $\mathrm{G} 1<\mathrm{G} 3 * *$ \\
\hline Perseverancia & $15.19(3.28)$ & $14.18(3.36)$ & $14.41(2.99)$ & 8.060 & .000 & 016 & $\mathrm{G} 1>\mathrm{G} 2 * * * \mathrm{G} 1>\mathrm{G} 3 * *$ \\
\hline Premeditación & $13.30(3.57)$ & $13.12(3.25)$ & $13.53(2.87)$ & 1.493 & .225 & .003 & \\
\hline Impulsividad Total & $50.54(9.87)$ & $53.88(9.55)$ & $53.15(8.34)$ & 10.726 & .000 & .021 & $\mathrm{G} 1<\mathrm{G} 2 * * * \mathrm{G} 1<\mathrm{G} 3 * *$ \\
\hline
\end{tabular}

(ítems $1,5,9,10,12,15$ y 20), Búsqueda de sensaciones (ítems 2, 7, 14 y 18), Perseverancia (ítems 6, 13, 16 y 17) y Premeditación (ítems 3, 4, $8,19)$.

La Tabla 2 indica los estadísticos descriptivos por sexos y para el grupo completo. Las diferencias entre medias fueron estadísticamente significativas únicamente en dos de las dimensiones:
Urgencia, con puntuaciones más elevadas para las chicas que para los chicos $(\mathrm{t}(2046)=-2.940$, $p=.003, \mathrm{~d}=-.13,95 \%$ IC $[-1.18,-.24])$, y Búsqueda de sensaciones, con niveles más altos para los varones que para las mujeres $(\mathrm{t}(2046)=7.203$, $p=.000, \mathrm{~d}=.32,95 \%$ IC $[1.02,1.77])$. No fueron significativas las diferencias para Perseverancia, Premeditación ni para Impulsividad Total. 
Posteriormente, se compararon los perfiles de impulsividad según la edad. Para ello se hicieron tres grupos de edad con los participantes. El primer grupo estuvo conformado por jóvenes de 11 y 12 años; el segundo, por aquellos de 13 y 14 años, y el tercero, por participantes de 15 a 17 años. La Tabla 3 resume estos resultados. Se aprecia que hay diferencias significativas en todos los factores al ser comparados por edades, aunque el tamaño del efecto es pequeño en todos los casos. Finalmente, se exploraron las diferencias por sexos en las dimensiones del UPPS en función de los rangos de edad establecidos. La Tabla 4 refleja los perfiles de los varones por rangos de edad, y la Tabla 5, los correspondientes perfiles de las mujeres. Mientras que los varones presentaron diferencias significativas por rangos de edad solamente en Urgencia, las mujeres mostraron diferencias significativas en los niveles de tres de las cuatro dimensiones del UPPS, excepto en Premeditación.

\section{Discusión}

Uno de los objetivos del estudio fue explorar las dimensiones de impulsividad, mediante una versión corta del UPPS, en una muestra amplia de adolescentes de ambos sexos, procedentes de América Latina y de España. Acorde con lo esperado, se replicó la estructura factorial de cuatro componentes en la muestra de este estudio, los cuales coinciden con los planteados por los autores del UPPS (Whiteside \& Lynam, 2001). No obstante, hubo tres ítems que migraron de su factor original: dos de ellos originales de Perseverancia saturaron más en Urgencia, y uno de Premeditación lo hizo en Perseverancia. A este respecto, d'Acremont y Van der Linden (2005) encontraron que la mayoría de ítems saturaron en sus respectivas dimensiones, aunque algunos de ellos lo hicieron de forma moderada o baja; otros en más de un factor, y otros migraron a otro factor, en el análisis por sexos. Con respecto a los valores de alfa de Cronbach, estos fueron próximos a los identificados por Keye et al. (2009), en Urgencia y en Búsqueda de sensaciones. No obstante, fueron más bajos en Perseverancia y en Premeditación, lo que hace que las conclusiones de estas dimensiones de carácter más cognitivo deban ser interpretadas con mayor cautela.

El segundo objetivo fue identificar si existen diferencias significativas por sexos en las dimensiones de impulsividad. Es así que Urgencia mostró niveles mayores en las mujeres que en los varones. La literatura existente ofrece resultados mixtos y escasos en torno a esta faceta de impulsividad; por un lado, trabajos en los que los niveles de Urgencia difieren de forma significativa entre varones y mujeres (d'Acremont \& Van der Linden, 2005), y otros en los que dichas diferencias no son significativas (Maneiro et al., 2017). Pese a lo anterior, las mujeres suelen presentar niveles más altos que los varones en esta dimensión. Un posible correlato de esta mayor tendencia de las mujeres de responder de forma impulsiva ante estados emocionales negativos procede del campo de la psicopatología, que arroja prevalencias mayores de mujeres con trastornos de ansiedad y con algunos trastornos depresivos, que se caracterizan por la experimentación intensa de emociones negativas (APA; DSM-5, 2013). Esta tendencia de las mujeres a presentar mayor psicopatología que los varones se evidencia también en estudios realizados con adolescentes (González, Ysern, Martorell, Matéu, \& Barreto, 2011).

Por otra parte, en consonancia con resultados procedentes de diversos trabajos, los niveles de Búsqueda de sensaciones fueron mayores para los varones que para las mujeres. A este respecto existe unanimidad, reflejada en estudios que han empleado el UPPS-P (Maneiro et al., 2017), así como otros instrumentos de medida (Pedersen et al., 2012; Romer \& Hennessy, 2007). Estos hallazgos sugieren que conforma un factor bastante estable, que sitúa a los varones como los más tendentes a experimentar situaciones interpretadas como gratificantes, aunque sean potencialmente riesgosas. Estudios procedentes de la psicología evolucionista, de las teorías de motivación, de la neuropsicología y de la psicopatología proporcionan posibles explicaciones de esta situación (Cross, Copping, \& Campbell, 2011). Estas dos dimensiones (Urgencia y Búsqueda de sensaciones) conforman los componentes emocionales o afectivos del UPPS, si bien están relacionados con emociones de distinta valencia, y con procesos de refuerzo 
negativo y positivo, respectivamente (Berg, Latzman, Bliwise, \& Lilienfeld, 2015); constituyen, además, dos rasgos de personalidad vinculados con síntomas internalizantes (la Urgencia) y externalizantes (la Búsqueda de sensaciones) (Quick \& Lakey, 2017).

Finalmente, en las facetas de impulsividad de carácter más cognitivo, que suponen autodisciplina (Perseverancia), y deliberación (Premeditación), no se encontraron diferencias significativas entre adolescentes hombres y mujeres, en este trabajo. Estos resultados van en la misma línea que otros realizados con adolescentes (d'Acremon \& Van der Linden, 2005; Maneiro et al., 2017), incluso al ser evaluados con otros instrumentos que miden facetas afines (Collado et al., 2014), lo cual sugiere cierto consenso científico al respecto, con independencia del instrumento empleado. La Premeditación y la Perseverancia están relacionadas con procesos auto-regulatorios y metas a largo plazo (Cyders \& Smith, 2007), a diferencia de la Urgencia, cuyo objetivo es la inmediata eliminación de la emoción negativa, mediante acciones no planificadas ni regidas por los mecanismos cognitivos de autorregulación (Berg et al., 2015). Por otra parte, si bien algunas actividades que se recogen en Búsqueda de sensaciones podrían suponer una minuciosa planificación y/o perseverancia para su realización, conviene mencionar que una de las primordiales diferencias entre las acciones que evalúan las dimensiones cognitivas frente a Búsqueda de sensaciones radica en el nivel de atractivo de las actividades y el potencial riesgo que suponen. Así, aquellas tareas evaluadas mediante las dimensiones de carácter cognitivo del UPPS suelen ser de tipo cotidiano, rutinario o poco novedoso a diferencia de las de Búsqueda de sensaciones.

El tercer objetivo fue analizar las trayectorias evolutivas por edades, desde la adolescencia temprana hasta la adolescencia media, pasando por una etapa de transición entre ambas adolescencias. Y el cuarto objetivo fue valorar si las trayectorias evolutivas por edades difieren al comparar a los participantes por sexos. En cuanto a la comparación por edades, al emplear el grupo completo, es decir, sin tener en cuenta la variable de género, se encontraron diferencias significativas en todas las dimensiones del UPPS.
La edad de 13 a 14 años se presenta como una etapa de transición entre la adolescencia temprana y la adolescencia media, y refleja cambios importantes en tres de las cuatro dimensiones evaluadas, a saber, en Urgencia, Perseverancia y Premeditación. De esta manera, se observa, en dicho rango de transición, el pico de Urgencia, situación que se mantiene tanto para hombres como para mujeres al dividir los análisis comparando por sexos, lo que sugiere que en esta etapa del desarrollo los jóvenes muestran una mayor tendencia que en otros períodos a reaccionar de forma impulsiva ante eventos que les generan emociones negativas. Solamente un estudio, hasta donde conocemos, exploró esta dimensión de impulsividad, desde una perspectiva de desarrollo, con una muestra de adolescentes de entre 11 y 16 años (Littlefield et al., 2016). Los resultados de dicho trabajo prospectivo mostraron cambios a nivel de puntuaciones medias; concretamente, el aumento significativo entre los 11 y los 13 años, y la posterior estabilización, reflejada en un incremento no significativo de los 13 a los 16 años. Estos hallazgos estarían en la misma línea de los encontrados en el presente estudio transversal, al hacer las comparaciones por edad y por género, en donde se aprecia que tras el pico en Urgencia hay un declive que, al parecer, es más abrupto para los varones que para las mujeres.

En lo que respecta a Perseverancia y Premeditación, ambas facetas presentaron los niveles más bajos a los 13 y 14 años, al analizar el grupo completo, lo que indicaría que en este período hay un decremento de dichas habilidades de control de la impulsividad, que son de carácter más cognitivo. No obstante, estas diferencias fueron significativas, en Perseverancia, solamente al comparar a los adolescentes tempranos con los otros dos grupos de adolescentes. Esta habilidad cognitiva disminuyó entre los participantes de mayores edades, perfil que se mantuvo en el análisis por sexos solamente en el grupo de las mujeres. Por el contrario, entre los varones, los niveles de Perseverancia fueron más estables a lo largo de los tres períodos de edad. La Premeditación, por otra parte, presentó los niveles más bajos en la etapa de transición y los más altos en la adolescencia media, aunque este perfil no se reflejó ni en varones ni en mujeres, al analizar por 
separado, lo que sugiere que en los períodos de la adolescencia evaluados hay un perfil similar de desarrollo en hombres y mujeres adolescentes, que indica niveles estables en esta variable. Estos hallazgos son complejos de interpretar dado que, hasta donde conocemos, no hay trabajos que evalúen las trayectorias evolutivas de estas dimensiones. La operacionalización de la variable "Impulsividad" empleada en estudios como el de Collado et al. (2014), Steinberg et al. (2008), Harden y Tucker-Drob (2011) y en el de Pedersen et al. (2012) podría sugerir ciertas similitudes con la faceta de Falta de premeditación del UPPS; no obstante, esta extrapolación ha de hacerse con cautela dado que se trata de variables diferentes. Pese a lo anterior, los resultados de Urgencia y de Perseverancia, al menos para el grupo de las mujeres, se podrían interpretar como aportaciones al modelo de sistemas duales (Casey et al. 2008) en cuanto a que se aprecia que, en el período de los 13 y 14 años, la Urgencia está más elevada que en las otras etapas, y es precisamente cuando más baja está la Perseverancia, lo que podría sugerir el carácter complementario de ambas facetas en la gestión de emociones.

En cuanto a Búsqueda de sensaciones, esta faceta mostró un incremento progresivo a medida que aumentaba la edad, al analizar el grupo completo; de modo que los participantes de entre 15 y 17 años fueron quienes presentaron mayores niveles en esta dimensión. Estos resultados van en la misma dirección que los reflejados en otros trabajos, que evidencian un aumento en los niveles de Búsqueda de sensaciones a lo largo de la adolescencia temprana y media (Collado et al., 2014; Littlefield et al., 2016), con su pico que oscila entre los 15 años (Steinberg et al., 2008) y los 17 años (Romer \& Hennessy, 2007). No obstante, al realizar el análisis por sexos, este patrón evolutivo se evidenció solamente en el grupo de las mujeres, mientras que los varones mostraron niveles más estables entre los tres rangos de edad. Resultados de otros trabajos van en consonancia con lo anterior, al señalar que las mujeres adolescentes presentan el pico en Búsqueda de sensaciones antes que los varones, concretamente a los 16 años frente a los 18.5 años de los hombres (Romer \& Hennessy, 2007), y al identificar incrementos menos pronunciados en los varones que en las mujeres (Littlefield et al., 2016).

Conocer las particularidades de mujeres y varones adolescentes en las diferentes dimensiones de impulsividad, en el transcurso de la adolescencia temprana a la adolescencia media, supone una aportación de este trabajo que podría ser utilizada para elaborar estrategias de prevención y de tratamiento de problemáticas o psicopatologías vinculadas con este multifacético constructo. Tener en cuenta la edad de 13 a 14 años como una etapa evolutiva de especial vulnerabilidad ante conductas impulsivas de tipo emocional (Urgencia), tanto para varones como para mujeres, sugiere la importancia de favorecer el entrenamiento de estrategias que permitan canalizar de maneras más adaptativas los estados afectivos negativos. La emoción positiva y el riesgo que supone la Búsqueda de sensaciones aporta información sobre los picos para cada sexo en la adolescencia, datos que son relevantes para comprender mejor la trayectoria de problemáticas como las adicciones (Marco \& Chóliz, 2017; Pérez-Fuentes et al., 2015) u otras conductas potencialmente peligrosas, como son las conductas antisociales (Martorell, González, Ordóñez, \& Gómez, 2011), que suelen establecerse en este rango de edad.

La etapa de transición entre la adolescencia temprana y la adolescencia media (los 13 y 14 años) supone también el momento evolutivo en el que se refleja un menor nivel de control cognitivo de la impulsividad (Perseverancia y Premeditación), con perfiles diferentes, según los sexos, para Perseverancia. Estos hallazgos podrían sugerir la importancia de un entrenamiento dirigido a incorporar estrategias de aprendizaje centradas en favorecer la motivación intrínseca por las tareas, así como técnicas de trabajo que fomenten el control cognitivo de la conducta. Hasta donde conocemos, este es el primer trabajo realizado con una muestra adolescente, procedente de Latinoamérica y de España, en el que se exploran estas dimensiones de impulsividad por sexo y por edades.

Este estudio presenta, no obstante, varias limitaciones. Al ser un estudio transversal, no permite valorar cambios intraindividuales, sino únicamente comparaciones entre puntuaciones medias intergrupales. Otra limitación es que la 
muestra, a pesar de su tamaño amplio, fue no probabilística y por conveniencia, lo que dificulta la generalización de estos resultados. Los datos fueron analizados sin tener en cuenta el país de procedencia, aspecto que sería importante abordar en trabajos posteriores de carácter transcultural. En ulteriores estudios se sugiere, además, explorar la dimensión de Urgencia positiva, a fin de analizar si su trayectoria a lo largo de la adolescencia es similar a la evidenciada en Urgencia negativa. Finalmente, las propiedades psicométricas de las subescalas de Premeditación y Perseverancia presentan niveles de fiabilidad aceptables, aunque bajos. Convendría, por tanto, en futuros trabajos explorar de modo más profundo estas dimensiones.

\section{Referencias}

American Psychiatric Association. (2013). Diagnostic and Statistical Manual of Mental Disorders, Fifth Edition. Washington, DC: American Psychiatric Association.

American Psychological Association. (2010). Publication Manual of the American Psychological Association, Sixth Edition. Washington, DC: American Psychological Association.

Berg, J. M., Latzman, R. D., Bliwise, N. G., \& Lilienfeld, S. O. (2015). Parsing the heterogeneity of impulsivity: A meta-analytic review of the behavioral implications of the UPPS for psychopathology. Psychological Assessment, $\quad 27$, 1129-1146. http://dx.doi.org/10.1037/pas0000111

Borges, A. M., Dahne, J., Lim, A. C., \& MacPherson, L. (2017). Negative affect mediates the relation between trait urgency and behavioral distress tolerance. Emotion, 17, 707-716. http://doi.org/10.1037/emo0000267

Burris, J. L., Riley, E., Puleo, G. E., \& Smith, G. T. (2017). A longitudinal study of the reciprocal relationship between ever smoking and urgency in early adolescence. Drug and Alcohol Dependence, 178, 519-526. http://dx.doi.org/10.1016/j.drugalcdep.2017.06.007

Casey, B. J., Getz, S., \& Galvan, A. (2008). The adolescent brain. Developmental Neuropsychology. 28, 62-77. doi: 10.1016/j.dr.2007.08.003

Collado, A., Felton, J. W., MacPherson, L., \& Lejuez, C. W. (2014). Longitudinal trajectories of sensation seeking, risk taking propensity, and impulsivity across early to middle adolescence. Addictive Behaviors, 39, 1580-1588.

http://dx.doi.org/10.1016/j.addbeh.2014.01.024

Cross, C. P., Copping, L. T., \& Campbell, A. (2011). Sex differences in impulsivity: A meta-analysis. Psychological Bulletin, 137, 97-130. doi:10.1037/a0021591

Cyders, M. A. (2015). The misnomer of impulsivity: Commentary on "choice impulsivity" and "rapid-response impulsivity" articles by Hamilton and colleagues. Personality Disorders: Theory, Research, and Treatment, 6, 204-205. doi:10.1037/per0000123

Cyders, M. A., Coskunpinar, A., \& VanderVeen, J. D. (2016). Urgency: A common transdiagnostic endophenotype for maladaptive risk taking. In V. Zeigler-Hill \& D. K. Marcus (Eds.), The dark side of personality: Science and practice in social, personality, and clinical psychology (pp. 157188). Washington, DC: American Psychological Association. http://dx.doi.org/10.1037/14854-009

Cyders, M. A., \& Smith, G. T. (2007). Moodbased rash action and its components: Positive and negative urgency. Personality and Individual Differences, 43, 839-850. doi:10.1016/j.paid.2007.02.008

Cyders, M. A., \& Smith, G. T. (2008). Emotionbased dispositions to rash action: Positive and negative urgency. Psychological Bulletin, 134, 807-828. http://doi.org/10.1037/a0013341

Cyders, M. A., Smith, G. T., Spillane, N. S., Fischer, S., Annus, A. M., \& Peterson, C. (2007). Integration of impulsivity and positive mood to predict risky behavior: Development and validation of a measure of positive urgency. Psychological Assessment, 19, 107-118. doi:10.1037/1040-3590.19.1.107

D’Acremont, M., \& Van der Linden, M. (2005). Adolescent impulsivity: Findings from a community sample. Journal of Youth and Adolescence, $\quad 34, \quad 427-435$. doi:10.1007/s10964-005-7260-1 
Durana, J. H., \& Barnes, P. A. (1993). A neurodevelopmental view of impulsivity and its relationship to the superfactors of personality. In The Impulsive Client; Theory, Research and Treatment, W.G. McCown, J.L. Johnson, and M.B. Shure, eds. (Washington, D.C.: American Psychological Association).

González, R., Ysern, L., Martorell, C., Matéu, C., \& Barreto, P. (2011). Relaciones entre psicopatología y apego en la adolescencia. Revista Iberoamericana de Diagnóstico y Evaluación - e Avaliação Psicológica, 29(1), 9-26.

Harden, K., \& Tucker-Drob, E. M. (2011). Individual differences in the development of sensation seeking and impulsivity during adolescence: Further evidence for a dual systems model. Developmental Psychology, 47, 739-746. doi:10.1037/a0023279

Keye, D., Wilhelm, O., \& Oberauer, K. (2009). Structure and correlates of the german version of the brief UPPS impulsive behavior scales. European Journal of Psychological Assessment, 25, 175-185. doi:http://dx.doi.org/10.1027/1015-5759.25.3.175

Littlefield, A. K., Stevens, A. K., Ellingson, J. M., King, K. M., \& Jackson, K. M. (2016). Changes in negative urgency, positive urgency, and sensation seeking across adolescence. Personality and Individual Differences, $\quad 90, \quad 332-337$. https://doi.org/10.1016/j.paid.2015.11.024

MacPherson, L., Magidson, J. F., Reynolds, E. K., Kahler, C. W. \& Lejuez, C. W. (2010). Changes in sensation seeking and risk-taking propensity predict increases in alcohol use among early adolescents. Alcoholism: Clinical and Experimental Research, 34, 1400-1408. doi:10.1111/j.1530-0277.2010.01223.x

Maneiro, L., Gómez-Fraguela, J. A., Cutrín, O., Romero, E. (2017). Impulsivity traits as correlates of antisocial behaviour in adolescents. Personality and Individual Differences, 104, 417-422. http://dx.doi.org/10.1016/j.paid.2016.08.045

Marco, C., \& Chóliz, M. (2017). Eficacia de las técnicas de control de la impulsividad en la prevención de la adicción a videojuegos. Terapia Psicológica, 35(1), 57-69.
Martorell, C., González, R., Ordóñez, A., \& Gómez, O. (2011). Estudio confirmatorio del cuestionario de conducta antisocial (CCA) y su relación con variables de personalidad y conducta antisocial. Revista Iberoamericana de Diagnóstico y Evaluación - e Avaliação Psicológica, 31(1), 97-114.

Patton, J. H., Stanford, M. S. \& Barratt, E. S. (1995). Factor structure of the Barratt impulsiveness scale. Journal of Clinical Psychology, 51(6), 768-774.

Pedersen, S. L., Molina, B. S., Belendiuk, K. A., \& Donovan, J. E. (2012). Racial differences in the development of impulsivity and sensation seeking from childhood into adolescence and their relation to alcohol use. Alcoholism: Clinical and Experimental Research, 36, 1794-1802. doi:10.1111/j.15300277.2012.01797.x

Pérez-Fuentes, M. C., Gázquez, J. J., Molero, M. M., Cardila, F., Martos, A., Barragán, A.B.,...Mercader, I. (2015). Impulsividad y consumo de alcohol y tabaco en adolescentes. European Journal of Investigation in Health, Psychology and Education, 5, 371-382. doi:10.1989/ejihpe.v5i3.139

Quick, E., \& Lakey, B. (2017). Social relations in sensation seeking and urgency: An SRM approach. Personality and Individual Differences, $\quad 111$ 37-45. http://dx.doi.org/10.1016/j.paid.2017.01.044

Romer, D., \& Hennessy, M. (2007). A biosocialaffect model of adolescent sensation seeking: The role of affect evaluation and peer-group influence in adolescent drug use. Prevention Science, 8, 89-101. doi:10.1007/s11121-007-0064-7

Sperry, S. H., Lynam, D. R., \& Kwapil, T. R. (2017). The convergence and divergence of impulsivity facets in daily life. Journal of Personality, 00, 1-12. doi:10.1111/jopy.12359

Stautz, K., \& Cooper, A. (2013). Impulsivityrelated personality traits and adolescent alcohol use: A meta-analytic review. Clinical Psychology Review, 33, 574-592. doi:10.1016/j.cpr.2013.03.003

Stautz, K., \& Cooper, A. (2014). Urgency traits and problematic substance use in adolescence: Direct effects and moderation of perceived peer use. Psychology of Addictive Behaviors, 28, 487-497. doi:10.1037/a0034346 
Steinberg, L., Albert, D., Cauffman, E., Banich, M., Graham, S., \& Woolard, J. (2008). Age differences in sensation seeking and impulsivity as indexed by behavior and selfreport: Evidence for a dual systems model. Developmental Psychology, 44, 1764-1778. doi:10.1037/a0012955

Stevens, J. R. (2017). Impulsivity: How time and risk influence decision

Making. Nebraska symposium on motivation, Volume 64. Lincoln, NE: Springer International Publishing. doi:10.1007/978-3-319-51721-6

Tomko, R. L., Prisciandaro, J. J., Falls, S. K., \& Magid, V. (2016). The structure of the UPPSR-Child impulsivity scale and its relations with substance use outcomes among treatment-seeking adolescents. Drug and Alcohol Dependence, 161, 276-283. http://dx.doi.org/10.1016/j.drugalcdep.2016.02.010

Whiteside, S. P., \& Lynam, D. R. (2001). The five factor model and impulsivity: Using a structural model of personality to understand impulsivity. Personality and Individual Differences, 30, 669-689. doi:10.1016/S0191-8869(00)00064-7

Zapolski, T. C., Stairs, A. M., Settles, R. F., Combs, J. L. \& Smith, G.T. (2010). The measurement of dispositions to rash action in children. Assessment, 17, 116-125. doi:10.1177/10731911093513 